\title{
Comparison of clinical features, molecular alterations, and prognosis in morphological subgroups of lung invasive mucinous adenocarcinoma
}

\author{
This article was published in the following Dove Press journal: \\ OncoTargets and Therapy \\ 18 November 2014 \\ Number of times this article has been viewed
}

\author{
Deng Cai ${ }^{1,2, *}$ \\ Hang $\mathrm{Li}^{1,2, *}$ \\ Rui Wang ${ }^{1,2}$ \\ Yuan $\mathrm{Li}^{2,3}$ \\ Yunjian Pan ${ }^{1,2}$ \\ Haichuan $\mathrm{Hu}^{1,2}$ \\ Yang Zhang ${ }^{1,2}$ \\ Ranxia Gongl,2 \\ Bin Pan ${ }^{1,2}$ \\ Yihua Sun ${ }^{1,2}$ \\ Haiquan Chen ${ }^{1,2}$ \\ 'Department of Thoracic Surgery, Fudan \\ University Shanghai Cancer Center, \\ Shanghai, People's Republic of China; \\ ${ }^{2}$ Department of Oncology, Shanghai \\ Medical College, Fudan University, \\ Shanghai, People's Republic of China; \\ ${ }^{3}$ Department of Pathology, Fudan \\ University Shanghai Cancer Center, \\ Shanghai, People's Republic of China \\ *These authors contributed equally to \\ this work
}

Correspondence: Haiquan Chen Department of Thoracic Surgery, Fudan University Shanghai Cancer Center, 270 Dong-An Road, Shanghai 200032,

People's Republic of China

Tel +86 2I 64175590

Fax +86 2I 6268 65II

Email hqchen I@yahoo.com

Yihua Sun

Department of Thoracic Surgery, Fudan University Shanghai Cancer Center, 270 Dong-An Road, Shanghai 200032, People's Republic of China

Tel +86 2I 64175590

Fax +86 2I 6268 65 II

Email sun_yihua76@hotmail.com
Purpose: We performed this analysis to distinguish the differences in two subtypes of lung invasive mucinous adenocarcinoma (IMA) with different kinds of morphological performances, in clinicopathological and molecular features, as well as prognosis.

Methods: On the basis of morphological performance, we divided lung IMAs into two subgroups, mucus-in-cell adenocarcinoma (MICA) and mucus-out-of-cell adenocarcinoma (MOCA). We investigated differences in clinicopathological characteristics, recurrence-free survival (RFS), overall survival (OS), and a spectrum of well-identified driver-gene mutations, including $E G F R, K R A S, H E R 2, B R A F, A L K, R O S 1$, and RET, between the two subgroups.

Results: Of 1,699 lung adenocarcinomas, 148 were identified as IMAs (97 MICAs and 51 MOCAs). The MICA patient group had significantly better RFS than did the MOCA group (39.4 months versus 33.0 months, respectively, log rank $P=0.020$ ) and significantly better OS (54.2 months versus 45.1 months, $\log$ rank $P=0.034$ ). There were no differences in RFS and OS between those with IMAs and those with mucus-negative adenocarcinomas. The frequency of the EGFR gene mutation was significantly higher in MOCAs than in MICAs $(P<0.001)$. In contrast, the KRAS gene had a significantly higher mutational frequency in MICAs $(P=0.01)$. MOCAs also had a significantly higher incidence of lymph-node metastasis $(P<0.05)$.

Conclusion: To our knowledge, this study represents the first comparison of clinical features, molecular alterations, and prognosis in morphological subgroups of lung IMAs. Clinical and pathological features in conjunction with molecular data indicate that IMA should be divided into different subgroups.

Keywords: lung, invasive mucinous adenocarcinoma, prognosis, driver mutations

\section{Introduction}

Non-small-cell lung cancer (NSCLC) is the leading cause of cancer-related death worldwide. ${ }^{1}$ The most common pathological subtype of diagnosed NSCLC is adenocarcinoma, which has an increasing prevalence. A new classification for lung adenocarcinoma was proposed by an international multidisciplinary expert panel from International Association for the Study of Lung Cancer/American Thoracic Society/ European Respiratory Society (IASLC/ATS/ERS) in $2011,{ }^{2}$ while the definition of mucinous bronchioloalveolar carcinoma is no longer used. Invasive mucinous adenocarcinoma (IMA) was recognized as a separate subtype of lung adenocarcinoma, which was supposed to contain components of columnar or goblet cells with abundant intracellular or extracellular mucus admixed with invasive adenocarcinoma patterns with 
stromal invasion. Compared with other lung adenocarcinoma subtypes, IMA has different immunohistochemical and molecular features, as well as a different prognosis..$^{3-5}$

Mucinous adenocarcinoma occurs in various organs and has two main kinds of histological appearance. In one, the tumor is composed of columnar or goblet cells with abundant intracellular mucus accumulation; in the other, the tumor has abundant extracellular mucus admixed with invasive adenocarcinoma patterns with stromal invasion. IMAs occurring in lung also share the above two kinds of histological appearance. We performed this retrospective study on surgically resected lung IMAs, with the aim to distinguish the differences in clinicopathological feature, molecular features, and prognosis between IMAs subgroups with these two kinds of morphological performance.

\section{Materials and methods}

\section{Patients and specimens}

From October 2007 to April 2013, patients with newly diagnosed primary lung cancer were consecutively enrolled. Inclusion criteria for this study included: 1) patients underwent complete resection of the primary lesion;2) pathological diagnosis of adenocarcinoma was reconfirmed by two pathologists (L Shen and Y Li); and 3) samples had a minimum of $50 \%$ tumor cells and sufficient corresponding normal tissue for mutational analysis. Patients who received any neoadjuvant chemotherapy or radiotherapy were excluded. Written informed consent was acquired from all the enrolled patients. This study was approved by our institutional review board.

\section{Clinical and pathological characteristics}

Clinicopathological data were collected for analyses of the association with IMA subtypes. Sex, smoking history, age at diagnosis, pathological cancer stage, histological type, and tumor differentiation were included. If a diagnosis of lung adenocarcinoma was made, histological subtypes according to the new IASLC/ATS/ERS multidisciplinary classification were collected. ${ }^{2}$ The abbreviation "MICA" was used to represent mucus-in-cell adenocarcinoma, while "MOCA" was used to define mucus-out-of-cell adenocarcinoma (Figure 1A, MICA; Figure 1B, MOCA). Tumors in which $>50 \%$ of cells contained abundant intracytoplasmic mucus were considered to be MICA, whereas tumors with few cells having intracytoplasmic mucus but with abundant extracellular mucus admixed with invasive adenocarcinoma patterns were considered to be MOCA. Lung adenocarcinoma without a mucinous component was defined as "mucus-negative". Patients were interviewed every 3 months after the date of diagnosis, either
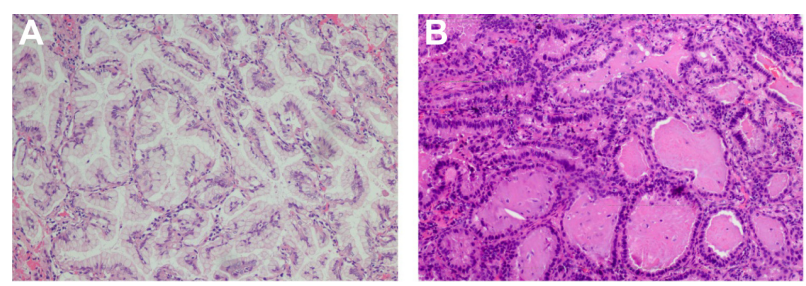

Figure I Different morphological appearances of lung invasive adenocarcinoma. Notes: (A) Mucus-in-cell adenocarcinoma $(>50 \%$ of tumor cells with abundant intracytoplasmic mucus; 10*10 magnification). (B) Mucus-out-of-cell adenocarcinoma (tumor cells with abundant extracellular mucus admixed with invasive adenocarcinoma patterns; $10 \times 10$ magnification).

in the clinic or by telephone, about disease recurrence and survival information. Recurrence-free survival (RFS) was calculated from the date of diagnosis to the date of recurrence or last-time follow-up. Overall survival (OS) was defined as the time elapsed between date of diagnosis to date of cancerrelated death or last-time follow-up.

\section{Mutation and gene-fusion analysis}

After the surgery, tissue samples were divided into two parts. One part was fixed using formalin and embedded with paraffin to make a pathological section; the other part was snap-frozen in liquid nitrogen at the time of resection and stored in liquid nitrogen. RNA and DNA were extracted from tumors, and distant histological normal lung after frozen specimens were dissected into TRIZOL (Life Technologies, CA, USA). Total RNA samples were reverse transcribed into complementary DNA.

EGFR (exons 18-21), KRAS (exons 2-3), HER2 (exons 18-21), BRAF (exons 11-15), PIK3CA (exons 9 and 20) were amplified by polymerase chain reaction (PCR) using complementary DNA. Amplified products were assessed using the Sanger direct-sequencing method ${ }^{6}$ in forward and reverse directions to detect mutations. All mutations were verified by analysis of an independent PCR isolate. ${ }^{7} \mathrm{~A}$ combined strategy of quantitative real-time PCR (qRT-PCR) and reverse-transcriptase PCR (RT-PCR) was performed to assess $A L K, R O S 1$, and RET fusion, with validation using fluorescent in situ hybridization. ${ }^{8}$

\section{Statistical analyses}

We adopted Pearson $\chi^{2}$ test or Fisher's exact test to assess the correlations between clinicopathological variables and IMA subtypes. Survival curves were made using the Kaplan-Meier method. Recurrence-free survival and overall survival were compared using log-rank test. Cox regression was performed to assess the effect of clinicopathological characteristics, including IMA subtypes, upon survival. 
The statistical analyses were performed using SPSS 16.0 for Windows (SPSS Inc., Chicago, IL, USA). All the tests were two-tailed, and statistical significance was set as $P<0.05$.

\section{Results}

Of 1,699 lung adenocarcinomas, 148 tumors turned out to be IMAs; of these, 97 were MICAs and 51 were MOCAs. There were 73 women and 75 men in this group, ranging in age at diagnosis from 23-84 years of age (median, 60 years). Nonsmokers comprised $67.6 \%$ (100 of 148) of patients. The number of patients with pathological stage I, II, and III cancer was 90,22 , and 36 , respectively (Table 1 ).

Through further comparison of the clinicopathological features between MICA and MOCA, we found that MOCAs had a significantly higher incidence of lymph-node metastasis ( $26.8 \%$ versus $43.1 \%, P<0.05$ ). There was no significant difference between the two categories in sex or age at diagnosis, nor in smoking history. The morphological performance

Table I Clinicopathological and molecular features of mucus-incell adenocarcinoma and mucus-out-of-cell adenocarcinoma

\begin{tabular}{|c|c|c|c|}
\hline Variables & $\operatorname{MICA}(n=97)$ & $\operatorname{MOCA}(n=5 I)$ & $P$-value \\
\hline \multicolumn{4}{|l|}{ Sex } \\
\hline Male & 45 & 30 & \multirow[t]{2}{*}{0.151} \\
\hline Female & 52 & 21 & \\
\hline \multicolumn{4}{|l|}{ Age, years } \\
\hline$\geq 60$ & 48 & 25 & \multirow[t]{2}{*}{0.957} \\
\hline$<59$ & 49 & 26 & \\
\hline \multicolumn{4}{|l|}{ Smoking history } \\
\hline Smoker & 27 & 21 & \multirow[t]{2}{*}{0.099} \\
\hline Never & 70 & 30 & \\
\hline \multicolumn{4}{|l|}{ Tumor size } \\
\hline$\leq 3 \mathrm{~cm}$ & 62 & 28 & \multirow[t]{2}{*}{0.286} \\
\hline$>3 \mathrm{~cm}$ & 35 & 23 & \\
\hline \multicolumn{4}{|l|}{ Cancer stage } \\
\hline I/II & 76 & 36 & \multirow[t]{2}{*}{0.296} \\
\hline III & 21 & 15 & \\
\hline \multicolumn{4}{|c|}{ Lymphovascular invasion } \\
\hline+ & 17 & 10 & \multirow[t]{2}{*}{0.099} \\
\hline- & 80 & 41 & \\
\hline \multicolumn{4}{|c|}{ Lymph node metastasis } \\
\hline No & 71 & 29 & \multirow[t]{2}{*}{0.044} \\
\hline $\mathrm{NI}-\mathrm{N} 3$ & 26 & 22 & \\
\hline \multicolumn{4}{|l|}{ Mutational status } \\
\hline EGFR mutation & 20 & 25 & $<0.001$ \\
\hline KRAS mutation & 22 & 3 & 0.01 \\
\hline HER2 mutation & 5 & 4 & 0.515 \\
\hline$B R A F$ mutation & 2 & 0 & 0.302 \\
\hline$A L K$ fusion & 16 & 4 & 0.206 \\
\hline ROSI fusion & 2 & I & 0.999 \\
\hline RET fusion & 3 & 3 & 0.415 \\
\hline Pan-negative & 27 & 11 & 0.407 \\
\hline
\end{tabular}

Abbreviations: MICA, mucus-in-cell adenocarcinoma; MOCA, mucus-out-of-cell adenocarcinoma. of mucus was not significantly correlated with tumor size, pathological stage, or lymphovascular invasion (Table 1).

In the 148 IMA cases, we detected 45 (30.4\%) EGFR mutations, 25 (16.9\%) KRAS mutations, nine (6.1\%) HER2 mutations, and two $B R A F(1.3 \%)$ mutations. We also found 20 (13.5\%) ALK fusions, six (4.1\%) RET fusions, and three (2.0\%) ROS1 fusions. All of these molecular alterations were mutually exclusive. Further, 27 (18.2\%) MICA and 11 (7.4\%) MOCA harbored none of the above molecular changes. The frequency of $E G F R$ gene mutation was significantly higher in MOCAs than in MICAs (49.1\% versus $20.6 \%, P<0.001)$. In contrast, the $K R A S$ gene had a significantly higher mutational frequency in MICAs $(22.7 \%$ versus $5.9 \%, P=0.01)$. The frequency of other well-identified driver-gene mutations did not significantly vary with different morphological performance of mucus (Table 1).

We further compared the clinical outcomes between 79 IMA and 439 consecutive mucus-negative lung adenocarcinomas, all of which were diagnosed from October 2007 to October 2011. We analyzed RFS and OS data from these two subgroups of patients. The median follow-up phase for the IMA group was 43.6 months and for the mucusnegative group was 48.5 months. The RFS and OS were not significantly different between IMA and mucus-negative lung adenocarcinoma groups (RFS: 37.2 months versus 38.8 months, respectively, log-rank $P=0.524$; OS: 49.5 months versus 63.5 months, respectively, log-rank $P=0.650$; Figure $2 \mathrm{~A}$ and $\mathrm{B})$. When IMAs were divided into MICA and MOCA subgroups and compared with the mucus-negative group, survival data showed a trend of difference between the three groups, but the results failed to reach statistical significance (RFS: MICA versus mucus-negative, log-rank $P=0.189$; RFS: MOCA versus mucus-negative, log-rank $P=0.648$; OS: MICA versus mucus-negative, log-rank $P=0.312$; OS: MOCA versus mucus-negative, log-rank $P=0.106$; Figure $2 \mathrm{C}$ and $\mathrm{D})$.

We then compared clinical outcomes between 38 MICAs and 41 MOCAs. Median follow-up time for the MICA group was 39.6 months and for the MOCA group was 41.5 months. Compared with the MICA group, the MOCA group had significantly worse RFS (39.4 months versus 33.0 months, respectively, log-rank $P=0.020)$ and OS (54.2 months versus 45.1 months, respectively, log-rank $P=0.034$ ) (Figure 2C and D). Smoking history was also correlated with worse RFS (31.4 months versus 39.9 months, respectively, $\log$-rank $P=0.021$ ) in IMAs, but no significant differences were found in OS (47.9 months versus 50.6 months, respectively, log-rank $P=0.47)$. Lymph node metastasis also 
A

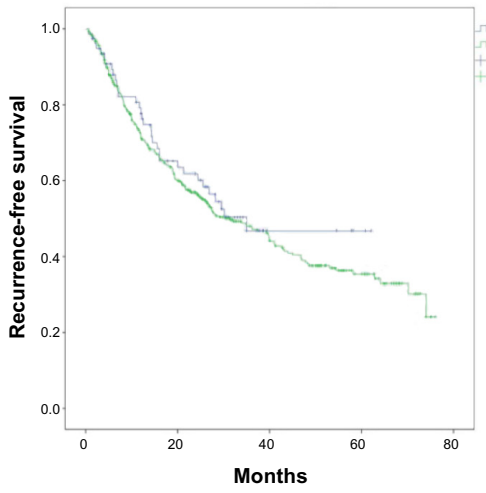

C

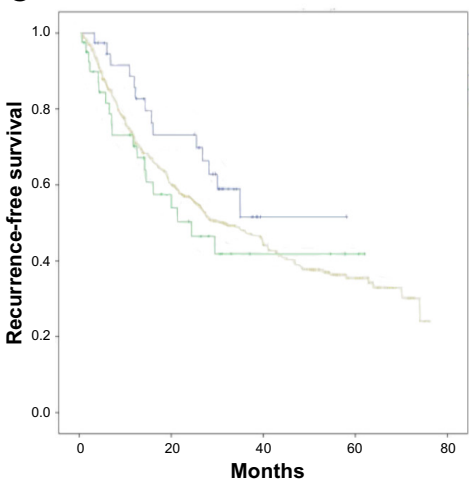

B

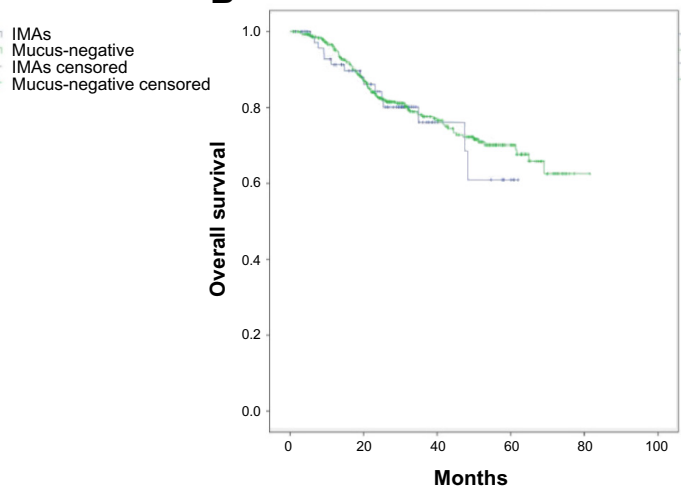

D

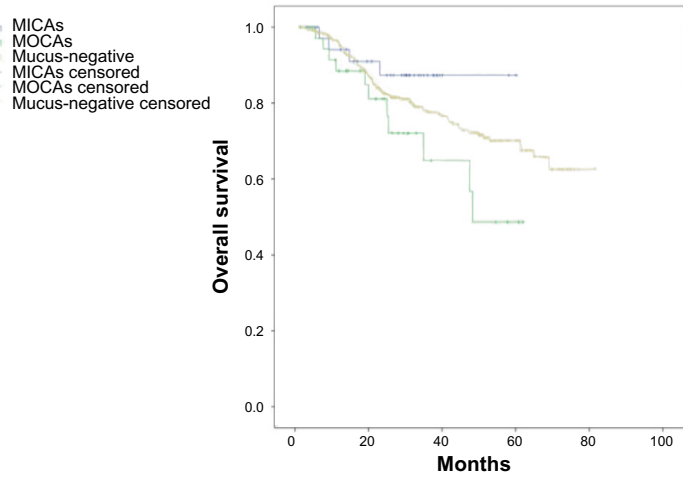

IMAs Mucus-negative Mucus-negative censored

Figure 2 Recurrence-free and overall survival.

Notes: (A) Recurrence-free survival of IMAs or mucus-negative adenocarcinomas. (B) Overall survival of IMAs or mucus-negative adenocarcinomas. (C) Recurrence-free survival of MICAs, MOCAs, or mucus-negative adenocarcinomas. (D) Overall survival of MICAs, MOCAs, or mucus-negative adenocarcinomas.

Abbreviations: IMA, invasive mucinous adenocarcinoma; MICA, mucus-in-cell adenocarcinoma; MOCA, mucus-out-of-cell adenocarcinoma.

predict worse RFS (31.6 months versus 41.0 months, log rank $P=0.007)$ and OS (44.3 months versus 53.0 months, $\log$ rank $P=0.018$ ) in 79 IMAs. Age at diagnosis, sex, lymphovascular invasion, and driver-gene mutational status had no significant correlations with RFS and OS. Multivariate analysis of survival significance was also performed, with age at diagnosis, sex, smoking history, mucus pattern, lymphovascular invasion, lymph node metastasis, and EGFR gene mutational status included. Lymph-node metastasis was the only independent factor of RFS (odd ratio $=3.24$, $95 \%$ confidence interval $=1.32-4.76, P<0.01)$, but had no significance for predicting OS.

\section{Discussion}

Since the appearance of the IASLC/ATS/ERS classification of lung adenocarcinoma in 2011, many studies have focused on the influence of pathological subtype on prognosis and molecular change. ${ }^{9-11}$ IMAs, which account for $2 \%-10 \%$ of lung adenocarcinoma cases in East Asia, Europe, and the United States, ${ }^{11-13}$ were considered to be more malignant than other common subtypes of lung adenocarcinoma, such as lepidic and acinar subtypes. ${ }^{14}$ To our knowledge, this is the first study to focus on the differences in prognosis and molecular alterations in IMA subgroups with different morphological features. Therefore, we performed this study to investigate if it is reasonable to divide invasive mucinous lung adenocarcinoma into separate parts.

Some previous studies demonstrated that IMAs correlated with worse prognosis. ${ }^{4,15}$ However, in some recent studies, IMA subtype failed to reach statistical significance as a worse prognosis predictor. ${ }^{16}$ Considering this discrepancy on the predictive value of IMA subtype on prognosis, we performed survival analysis between IMA patients and mucus-negative patients. Our results indicated that the IMA patients did not have a worse prognosis than mucus-negative patients do. Dividing IMAs into two subgroups by different morphological performance and comparing all groups, we found that patients with MICA showed a potential trend of better prognosis than did mucus-negative patients, while MOCAs seemed to have the worst prognosis of all three subgroups. Our results indicated that IMA subtype alone may not be an adequate predictor for worse prognosis. Different morphological performances may have potential predictive value on clinical outcome. 
We also observed distinct prognoses between IMAs with different morphological performance. Patients with MICA had a better RFS and OS than did their counterparts with MOCA. Lymph-node metastasis also occurred more frequently in patients with MOCA. To our knowledge, no similar study has revealed different clinical outcomes between invasive mucinous lung adenocarcinoma subgroups with different mucus distributions. Mucinous adenocarcinomas in other organs are also associated with lymph-node metastasis and worse prognosis, ${ }^{17-20}$ and mucus is considered to play a critical role in the development of cancer. ${ }^{21}$ Thus, we suspect that the common phenomenon is related to the same mucinous component in different cancers. When the mucus is out of the cell, the tumor may have aggressive biological behavior. However, further study is needed to form a more reliable conclusion.

Lung IMA was reported to be associated with higher frequency of $K R A S$ mutation and resistance to $E G F R$ tyrosine kinase inhibitor. ${ }^{12,22,23}$ In our series, the frequency of $E G F R$ and $K R A S$ mutation was $30.4 \%$ and $16.9 \%$, respectively. Also, a higher prevalence of $A L K$ fusion was observed in IMAs than in unselected lung adenocarcinoma. ${ }^{24}$ We further analyzed the association between morphological feature and molecular alteration, finding that the frequency of $E G F R$ mutation was remarkably higher in MOCAs than in MICAs and that KRAS mutation significantly prevailed in MICAs. These findings suggest that there is a distinct difference in molecular change in these two subgroups of IMA, and that MICAs and MOCAs are two different kinds of adenocarcinoma. Therefore, more accurate distinction is required to be made in IMAs.

The weakness of our work was that this was a retrospective study. The clinical outcomes had limited significance, because some of these patients had different postoperative treatments. Meanwhile, a sample size of 148 patients was too small to prove the differences between IMA subgroups with different morphological features. This issue should be confirmed by further research with larger samples.

\section{Conclusion}

We found various subtypes of lung IMAs. There are distinct morphological performances, driver-gene mutation distributions, and clinical outcomes in the two subgroups of patients. A more precise definition of IMA should be taken into consideration in the future.

\section{Acknowledgments}

The authors acknowledge financial support from the Key Construction Program of the National "985" Project
(Grant No 985III-YFX0102), the National Natural Science Foundation of China (Grants No 81172218 and 81101761), the Science and Technology Commission of Shanghai Municipality (Program of Shanghai Subject Chief Scientist; Grant No 12XD1402000), the Foundation of Shanghai Health Administration (Grant No 20114206), and the Shanghai Hospital Development Center (Grant No SHDC12012308). The authors thanked Yuanshan Fu for language proofreading.

\section{Disclosure}

The authors declare that no benefits in any form have been received or will be received from a commercial party related directly or indirectly to the subject of this article. The authors report no other conflicts of interest in this work.

\section{References}

1. Jemal A, Bray F, Center MM, Ferlay J, Ward E, Forman D. Global cancer statistics. CA Cancer J Clin. 2011;61(2):69-90.

2. Travis WD, Brambilla E, Noguchi M, et al. International association for the study of lung cancer/american thoracic society/european respiratory society international multidisciplinary classification of lung adenocarcinoma. J Thorac Oncol. 2011;6(2):244-285.

3. Zhang Y, Sun Y, Xiang J, Zhang Y, Hu H, Chen H. A clinicopathologic prediction model for postoperative recurrence in stage Ia non-small cell lung cancer. J Thorac Cardiovasc Surg. 2014;148(4):1193-1199.

4. Casali C, Rossi G, Marchioni A, et al. A single institution-based retrospective study of surgically treated bronchioloalveolar adenocarcinoma of the lung: clinicopathologic analysis, molecular features, and possible pitfalls in routine practice. J Thorac Oncol. 2010;5(6):830-836.

5. Goldstein NS, Thomas M. Mucinous and nonmucinous bronchioloalveolar adenocarcinomas have distinct staining patterns with thyroid transcription factor and cytokeratin 20 antibodies. Am J Clin Pathol. 2001;116(3):319-325.

6. Sanger F, Nicklen S, CoulsonAR. DNA sequencing with chain-terminating inhibitors. Proc Natl Acad Sci U S A. 1977;74(12):5463-5467.

7. Li H, Pan Y, Li Y, et al. Frequency of well-identified oncogenic driver mutations in lung adenocarcinoma of smokers varies with histological subtypes and graduated smoking dose. Lung Cancer. 2013;79(1): 8-13.

8. Wang R, Hu H, Pan Y, et al. RET fusions define a unique molecular and clinicopathologic subtype of non-small-cell lung cancer. J Clin Oncol. 2012;30(35):4352-4359.

9. Hung JJ, Jeng WJ, Chou TY, et al. Prognostic value of the new International Association for the Study of Lung Cancer/American Thoracic Society/European Respiratory Society lung adenocarcinoma classification on death and recurrence in completely resected stage I lung adenocarcinoma. Ann Surg. 2013;258(6):1079-1086.

10. Russell PA, Barnett SA, Walkiewicz M, et al. Correlation of mutation status and survival with predominant histologic subtype according to the new IASLC/ATS/ERS lung adenocarcinoma classification in stage III (N2) patients. J Thorac Oncol. 2013;8(4):461-468.

11. Warth A, Muley T, Meister M, et al. The novel histologic International Association for the Study of Lung Cancer/American Thoracic Society/ European Respiratory Society classification system of lung adenocarcinoma is a stage-independent predictor of survival. J Clin Oncol. 2012;30(13):1438-1446.

12. Tsuta K, Kawago M, Inoue E, et al. The utility of the proposed IASLC/ ATS/ERS lung adenocarcinoma subtypes for disease prognosis and correlation of driver gene alterations. Lung Cancer. 2013;81(3): 371-376. 
13. Yoshizawa A, Motoi N, Riely GJ, et al. Impact of proposed IASLC/ATS/ ERS classification of lung adenocarcinoma: prognostic subgroups and implications for further revision of staging based on analysis of 514 stage I cases. Mod Pathol. 2011;24(5):653-664.

14. Cadranel J, Quoix E, Baudrin L, et al; IFCT-0401 Trial Group. IFCT0401 Trial: a phase II study of gefitinib administered as first-line treatment in advanced adenocarcinoma with bronchioloalveolar carcinoma subtype. J Thorac Oncol. 2009;4(9):1126-1135.

15. Russell PA, Wainer Z, Wright GM, Daniels M, Conron M, Williams RA. Does lung adenocarcinoma subtype predict patient survival?: A clinicopathologic study based on the new International Association for the Study of Lung Cancer/American Thoracic Society/European Respiratory Society international multidisciplinary lung adenocarcinoma classification. J Thorac Oncol. 2011;6(9):1496-1504.

16. Westaway DD, Toon CW, Farzin M, et al. The International Association for the Study of Lung Cancer/American Thoracic Society/European Respiratory Society grading system has limited prognostic significance in advanced resected pulmonary adenocarcinoma. Pathology. 2013;45(6):553-558.

17. Consorti F, Lorenzotti A, Midiri G, Di Paola M. Prognostic significance of mucinous carcinoma of colon and rectum: a prospective case-control study. J Surg Oncol. 2000;73(2):70-74.

18. Enciu M, Aşchie M, Deacu M, Poinăreanu I. Morphological characteristics of a mucinous adenocarcinoma of the prostate: differential diagnosis considerations. Rom J Morphol Embryol. 2013;54(1): 191-194.
19. Kunisaki C, Akiyama H, Nomura M, et al. Clinicopathologic characteristics and surgical outcomes of mucinous gastric carcinoma. Ann Surg Oncol. 2006;13(6):836-842.

20. Kawamura H, Kondo Y, Osawa S, et al. A clinicopathologic study of mucinous adenocarcinoma of the stomach. Gastric Cancer. 2001;4(2): 83-86.

21. Beatty PL, Narayanan S, Gariépy J, Ranganathan S, Finn OJ. Vaccine against MUC1 antigen expressed in inflammatory bowel disease and cancer lessens colonic inflammation and prevents progression to colitis-associated colon cancer. Cancer Prev Res (Phila). 2010;3(4): 438-446.

22. Yoshizawa A, Sumiyoshi S, Sonobe M, et al. Validation of the IASLC/ATS/ERS lung adenocarcinoma classification for prognosis and association with EGFR and KRAS gene mutations: analysis of 440 Japanese patients. J Thorac Oncol. 2013;8(1):52-61.

23. Wislez M, Antoine M, Baudrin L, et al. Non-mucinous and mucinous subtypes of adenocarcinoma with bronchioloalveolar carcinoma features differ by biomarker expression and in the response to gefitinib. Lung Cancer. 2010;68(2):185-191.

24. Soda M, Choi YL, Enomoto M, et al. Identification of the transforming EML4-ALK fusion gene in non-small-cell lung cancer. Nature. 2007; 448(7153):561-566.
OncoTargets and Therapy

\section{Publish your work in this journal}

OncoTargets and Therapy is an international, peer-reviewed, open access journal focusing on the pathological basis of all cancers, potential targets for therapy and treatment protocols employed to improve the management of cancer patients. The journal also focuses on the impact of management programs and new therapeutic agents and protocols on

\section{Dovepress}

patient perspectives such as quality of life, adherence and satisfaction The manuscript management system is completely online and includes a very quick and fair peer-review system, which is all easy to use. Visit http://www.dovepress.com/testimonials.php to read real quotes from published authors. 